\title{
The Role of System-Specific Molecular Chaperones in the Maturation of Molybdoenzymes in Bacteria
}

\author{
Meina Neumann and Silke Leimkühler \\ Department of Molecular Enzymology, Institute of Biochemistry and Biology, University of Potsdam, 14476 Potsdam, Germany \\ Correspondence should be addressed to Silke Leimkühler, sleim@uni-potsdam.de
}

Received 30 June 2010; Accepted 31 August 2010

Academic Editor: Emil Pai

Copyright ( $\odot 2011$ M. Neumann and S. Leimkühler. This is an open access article distributed under the Creative Commons Attribution License, which permits unrestricted use, distribution, and reproduction in any medium, provided the original work is properly cited.

\begin{abstract}
Biogenesis of prokaryotic molybdoenzymes is a complex process with the final step representing the insertion of a matured molybdenum cofactor (Moco) into a folded apoenzyme. Usually, specific chaperones of the XdhC family are required for the maturation of molybdoenzymes of the xanthine oxidase family in bacteria. Enzymes of the xanthine oxidase family are characterized to contain an equatorial sulfur ligand at the molybdenum center of Moco. This sulfur ligand is inserted into Moco while bound to the XdhC-like protein and before its insertion into the target enzyme. In addition, enzymes of the xanthine oxidase family bind either the molybdopterin (Mo-MPT) form of Moco or the modified molybdopterin cytosine dinucleotide cofactor (MCD). In both cases, only the matured cofactor is inserted by a proofreading process of XdhC. The roles of these specific XdhClike chaperones during the biogenesis of enzymes of the xanthine oxidase family in bacteria are described.
\end{abstract}

\section{Introduction}

Molybdenum is a transition metal that is incorporated as a biologically active cofactor (molybdenum cofactor, Moco) in a class of widely distributed proteins collectively known as molybdoenzymes [1]. Moco is associated with a wide range of redox enzymes and is found in most organisms from bacteria to humans. The metal in Moco is coordinated to a pterin derivative called molybdopterin to form the molybdenum-containing molybdopterin (MoMPT) cofactor [2]. A wide variety of transformations are catalyzed by these enzymes at carbon, sulfur, and nitrogen atoms, which include the transfer of an oxygen group or two electrons to or from the substrate. The mononuclear molybdenum enzymes are categorized on the basis of the structures of their molybdenum centers, dividing them into three families, each with a distinct active site structure and a distinct type of reaction catalyzed: the xanthine oxidase family, the sulfite oxidase family, and the DMSO reductase family [1] (Figure 1). The xanthine oxidase family is characterized by an $\mathrm{LMo}^{V I} \mathrm{OS}(\mathrm{OH})$ core in the oxidized state, with one equivalent of the pterin cofactor (designated L) coordinated to the metal. These enzymes (including xanthine dehydrogenase $[\mathrm{XDH}]$ and xanthine oxidase $[\mathrm{XO}]$ ) typically catalyze the hydroxylation of carbon centers [1]. Enzymes of the sulfite oxidase family coordinate a single equivalent of the pterin cofactor with an $\mathrm{LMo}^{V I} \mathrm{O}_{2}$ (S-Cys) core in its oxidized state (the cysteine ligand is provided by the polypeptide) [1]. Members of this family (including sulfite oxidase and plant nitrate reductase) catalyze the transfer of an oxygen atom either to or from the substrate. The DMSO reductase family is diverse in both structure and function, but all members have two equivalents of the pterin cofactor bound to the metal. The molybdenum coordination sphere is usually completed by a single $\mathrm{M}=\mathrm{O}$ group with a sixth ligand in the $\mathrm{L}_{2} \mathrm{M}^{V I} \mathrm{O}(\mathrm{X})$ core [1]. The sixth ligand, $\mathrm{X}$, can be a serine, a cysteine, a selenocysteine, or a hydroxide and/or water molecule. The reactions catalyzed by members of this family frequently involve oxygen-atom transfer, but dehydrogenation reactions also occur. While in bacteria, molybdoenzymes of all three families are present, eukaryotes only harbour molybdoenzymes of the xanthine oxidase or sulfite oxidase family. In bacteria, the basic form of Mo-MPT is generally modified by the attachment of CMP or GMP to Mo-MPT, forming the molybdopterin cytosine dinucleotide cofactor (MCD) or the bis-molybdopterin 
guanine dinucleotide cofactor (bis-MGD) (Figure 1) [3]. While the bis-MGD form of Moco is exclusively distributed in enzymes of the DMSO reductase family, the MCD cofactor was identified in bacterial enzymes of the xanthine oxidase family. In this family, enzymes contain either Mo-MPT or MCD with an equatorial sulfur ligand coordinated to the molybdenum center [4].

Enzymes of the xanthine oxidase family are the best characterized mononuclear molybdenum-containing enzymes. With a few exceptions, they catalyze the hydroxylation of different types of substrates according to the following reaction:

$$
\mathrm{RH}+\mathrm{H}_{2} \mathrm{O}=\mathrm{ROH}+2 \mathrm{H}^{+}+2 \mathrm{e}^{-} .
$$

This reaction occurs at the molybdenum center, which is, after interaction with substrate, reduced from $\mathrm{Mo}(\mathrm{VI})$ to $\mathrm{Mo}(\mathrm{IV})$. The two reducing equivalents generated in the course of the reaction are then transferred to an external electron acceptor by means of an electron transfer process mediated by other redox cofactors present in the structure of the protein. The crystal structure of the eight members of this family has been reported which are Desulfovibrio gigas aldehyde oxidoreductase (DgAOR) [5], Desulfovibrio desulfuricans aldehyde oxidoreductase (DdAOR) [6], Bos taurus xanthine oxidoreductase (bXOR) [7], Rhodobacter capsulatus xanthine dehydrogenase (RcXDH, Figure 2) [8], Pseudomonas putida Quinoline 2-oxidoreductase (PpQOR) [9], Thauera aromatica 4-hydroxylbenzoyl-CoA reductase (Ta 4-HBCR) [10] and the carbon monoxide dehydrogenase (CODH) from Oligotropha carboxidovorans [11] and Hydrogenophaga pseudoflava [12]. While bXOR and RcXDH contain Mo-MPT, all other crystallized bacterial enzymes were identified to bind MCD [13]. The majority of these enzymes (with the exception of 4-Hydroxybenzoyl-CoA reductase) are complex metalloflavoproteins that contain two nonidentical [2Fe2S] clusters, FAD, and the Moco as catalytically acting units (Figure 2) [14]. By contrast, CODH, which catalyzes a different reaction to that shown in (1), the conversion of $\mathrm{CO}$ to $\mathrm{CO}_{2}$ without cleavage of the $\mathrm{CH}$ bond, shows an active site that has never been observed in molybdoenzymes containing the pterin cofactor [15]. This comprises a dinuclear heterometal $\left[\mathrm{CuSMoO}_{2}\right]$ cluster in which $\mathrm{Mo}$ and $\mathrm{Cu}$ ions are bridged by a sulfur ligand. $\mathrm{CO}$ oxidation is a source of energy for a wide diversity of prokaryotes and is an important process within the global carbon cycle. The enzyme CODH catalyzes the oxidation of $\mathrm{CO}$ and water to produce carbon dioxide, two electrons, and two protons [15]. The electrons are transferred to an electron transfer chain and used to generate a proton gradient across the membrane.

Xanthine oxidoreductase (XOR; which can be splitted into XDH, EC 1.17.1.4, and XO, EC 1.17.3.2) and aldehyde oxidase (AO; EC 1.2.3.1) are the best characterized members of the xanthine oxidase family $[14,16]$. XOR has been the object of many reports and has long been recognized as the key enzyme in the catabolism of purines (Figure 2), oxidizing hypoxanthine to xanthine and xanthine to the terminal catabolite, uric acid, with the concomitant reduction of
$\mathrm{NAD}^{+}(\mathrm{XDH})$ or $\mathrm{O}_{2}(\mathrm{XO})[17,18]$. XORs are of considerable medical interest, since this enzyme system in humans is implicated in gout and hyperuricemia, and its activity is responsible for postischaemic reperfusion injury. In bacteria, uric acid in most cases is further degraded to urea which is hydrolyzed to $\mathrm{NH}_{4}^{+}$and $\mathrm{CO}_{2}$ and thus can serve as a nitrogen and/or carbon source [19]. It was shown that $R$. capsulatus has the ability to utilize purines as sole nitrogen source [19]. While the biochemical function of XOR is well established, the biochemical and physiological function of $\mathrm{AO}$ is still largely obscure. The overall level of similarity between $\mathrm{AO}$ and XOR proteins is approximately 50\%, which clearly indicates that the two proteins originated from a common ancestral precursor [14]. In humans, single monogenic deficits for any $\mathrm{AO}$ have not been described yet. $\mathrm{AO}$ is characterized by broad substrate specificity, and this makes it an important enzyme in the metabolism of drugs and xenobiotics. The physiological role of DgAOR was proposed to be linked to the degradation of polyglucose [5]. Also, in contrast to the other molybdo-flavoenzymes, DgAOR lacks the FAD domain [5]. Bacterial AOR can have a preference for aromatic aldehydes, as shown for the aldehyde oxidoreductase PaoABC from E. coli [20]. The physiological role of PaoABC in E. coli has been suggested to catalyze the detoxification of aromatic aldehydes to their corresponding less toxic acids in the periplasm [20].

The crystal structures of several molybdoenzymes revealed that Moco is deeply buried inside the proteins, at the end of a funnel-shaped passage giving access only to the substrate [21] (Figure 2). This implied the requirement of specific chaperones for each molybdoenzyme, to facilitate the insertion of Moco. For R. capsulatus XDH, the XdhC protein has not only been identified to be involved in Moco binding and formation of the terminal sulfur ligand, but was also shown to be involved in the final folding and maturation of apo-XDH after Moco insertion [22]. In prokaryotes, a number of specific chaperones were identified, also for members of the DMSO reductase family, like TorD for $E$. coli and trimethylamine oxide (TMAO) reductase (TorA) or NarJ for E. coli nitrate reductase A (NarGHI) [23-26]. This paper will focus on the molecular chaperones involved in the maturation of enzymes from the xanthine oxidase family in bacteria. At the end, a short comparison to the eukaryotic system is presented.

\section{In Bacterial Genomes, Structural Genes Encoding for Members of the Xanthine Oxidase Family and Molecular Chaperones Are Clustered}

For $R$. capsulatus $\mathrm{XDH}$, two genes, $x d h A$ and $x d h B$, encode the polypeptides of the active enzyme [19]. XdhA was shown to bind the FAD cofactor and two [2Fe2S] clusters, and $\mathrm{XdhB}$ was shown to bind Mo-MPT (Figure 2) [8]. Immediately downstream of $x d h B$, a third gene was identified, designated $x d h C$, which is cotranscribed with $x d h A B$ (Figure 3). Interposon mutagenesis revealed that the $x d h C$ gene product is required for XDH activity [22]. However, 


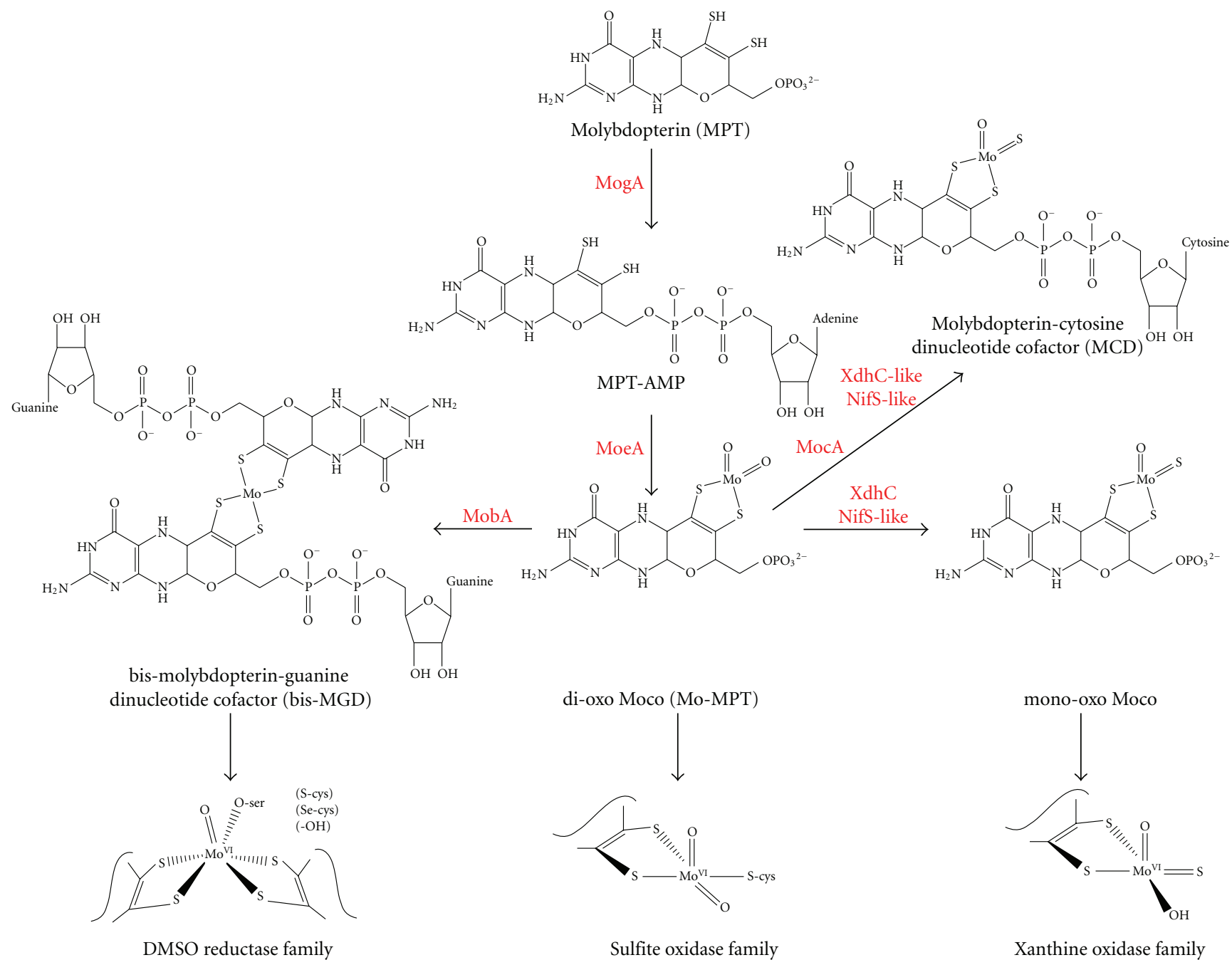

Figure 1: The biosynthesis of Moco and the families of molybdoenzymes. Shown is a scheme of the biosynthetic pathway of Moco from MPT in bacteria. The proteins involved in the reactions are colored red. In bacteria, Mo-MPT can be further modified by the MobA function which attaches GMP to the phosphate group of MPT, forming MGD, and two equivalents of MGD are bound to molybdenum, forming the so-called bis-MGD cofactor. For enzymes of the xanthine oxidase family, Mo-MPT or MCD (which is synthesized by the MocA protein by the attachment of CMP to Mo-MPT) can be modified by the replacement of one oxo-ligand by a sulfur ligand while forming the mono-oxo Moco. This reaction is catalyzed by a NifS-like protein in bacteria. The three molybdenum-containing enzyme families are divided into the DMSO reductase, the sulfite oxidase, and the xanthine oxidase families according to their active-site structures. The molybdenum center is shown in its oxidized state as $\mathrm{Mo}^{\beta}$. Moco is the general term for all different variants of the cofactor.

$\mathrm{XdhC}$ is not a subunit of active $\mathrm{XDH}$, which forms an $(\alpha \beta)_{2}$ heterodimer in R. capsulatus (Figure 2). It was shown that $\mathrm{XdhC}$ neither is a transcriptional regulator for $x d h$ gene expression nor influences XDH stability. The absence of Mo-MPT from $\mathrm{XDH}$ isolated from an $R$. capsulatus $x d h C$ mutant strain indicated that $\mathrm{XdhC}$ might be a specific chaperone facilitating the insertion of Mo-MPT into XDH [22].

Genes similar to $R$. capsulatus $x d h C$ have also been identified in a number of other prokaryotes. In some cases, a similar operon organization compared to the one present for $R$. capsulatus $\mathrm{XDH}$ was identified. However, so far, $R$. capsulatus $\mathrm{XdhC}$ is the only member of this family which has been characterized both on the genetic level and on the protein level [28].
Pseudomonas aeruginosa contains an $x d h$ operon consisting of the genes $x d h A B C$, like the one identified in R. capsulatus (Figure 3 ). Here, also $x d h A B$ code for the structural genes of XDH with a similar subunit composition as identified for $R$. capsulatus $\mathrm{XDH}$. An essential role of $P$. aeruginosa $\mathrm{XdhC}$ for $\mathrm{XDH}$ has not been demonstrated yet, for example, by interposon mutagenesis or on the purified proteins. However, for $P$. aeruginosa XdhC, it was shown that coexpression with the $x d h A B$ structural genes from Comamonas acidovorans in a heterologous system in E. coli resulted in the production of an active XDH [29]. In the absence of $P$. aeruginosa XdhC, a lower Mo-MPT content was identified in the purified protein [29]. This suggested a similar role for P. aeruginosa XdhC in Mo-MPT insertion and $\mathrm{XDH}$ maturation as shown for R. capsulatus XdhC. 


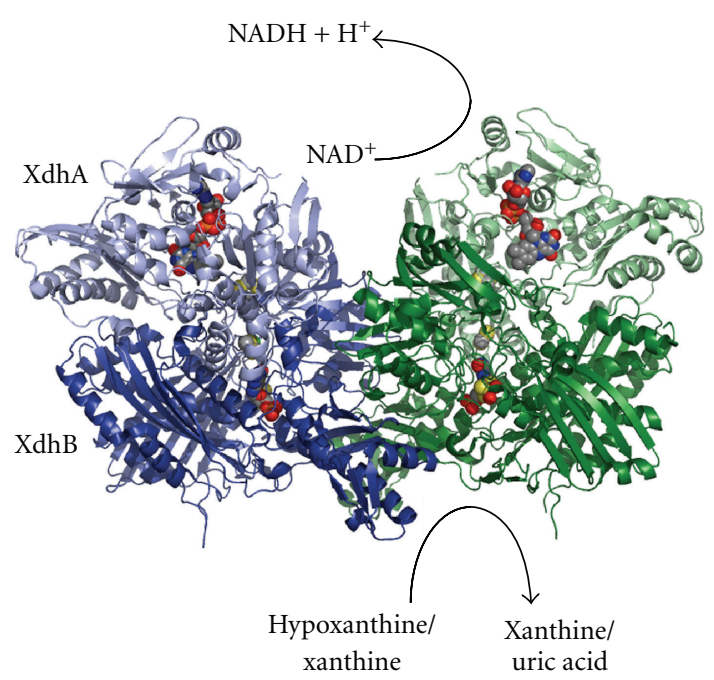

(a)

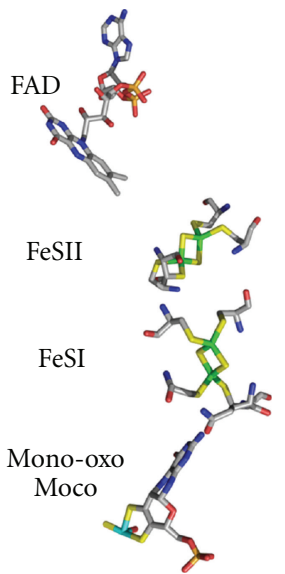

(b)

FIGURE 2: Overall structure and cofactor arrangement of $R$. capsulatus XDH. R. capsulatus XDH forms an $(\alpha \beta)_{2}$ heterotetramer. The XdhA subunits are drawn in light green and light blue and the XdhB subunits in dark green and dark blue. The [2Fe-2S] and FAD cofactors of XdhA and the Moco of XdhB are shown as space-filling models. The Moco is deeply buried in the XdhB subunit being only accessible through a substrate-binding channel. Also shown is the coordination of Moco and FeSI, FeSII and FAD at the active site of R. capsulatus XDH and the reaction catalyzed by XDH. The structures were generated using the coordinates from the Protein Data Bank (accession number 1JRO).
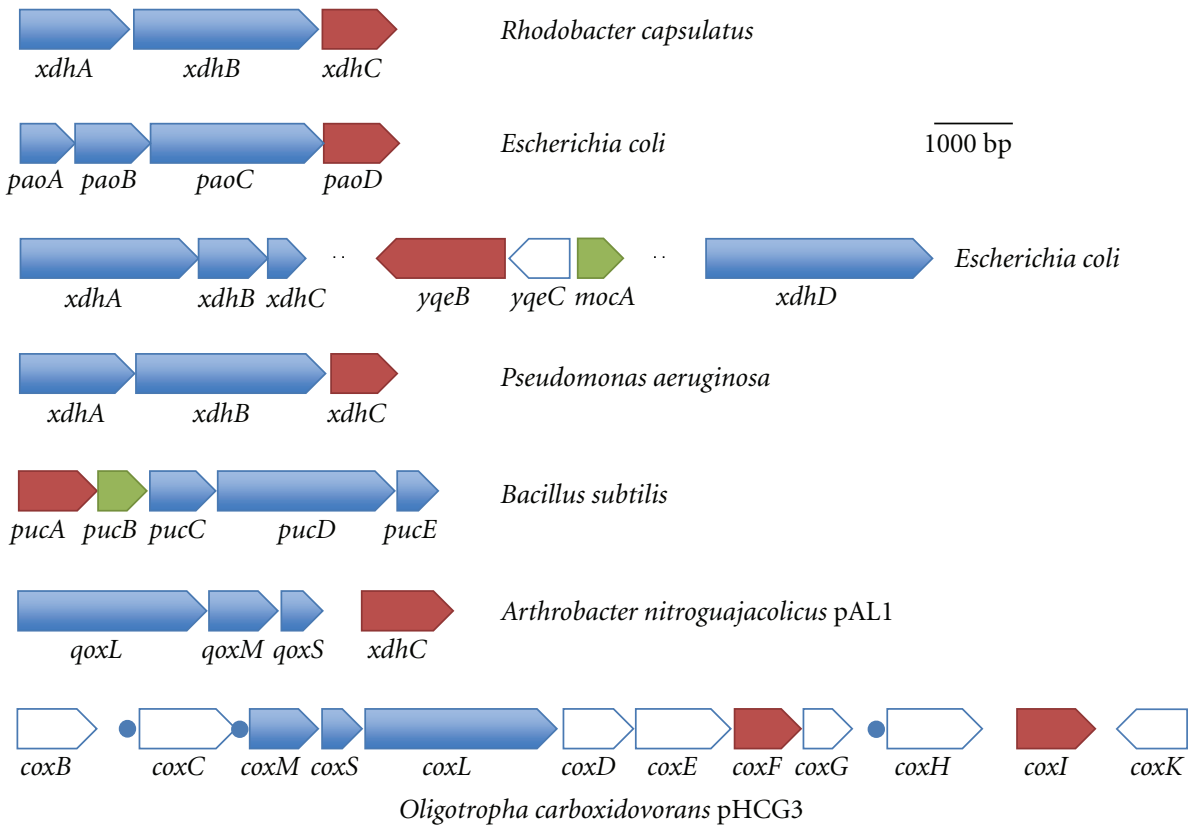

FIGURE 3: Schematic overview and organisation of operons including $x d h C$-like genes. Shown is the operon organization of different operons encoding for molybdoenzymes of the xanthine oxidase family of different bacteria. Operons of characterized molybdoenzymes are marked in blue, highlighted in red are the predicted genes encoding $\mathrm{XdhC}$ homologues, marked in green are genes for putative MocA-homologues, and marked in white are open reading frames encoding proteins with different (or not assigned) roles. In the operon structure encoding for the $\mathrm{CODH}$, blue dots indicate predicted promoter sequences as published by Santiago et al. [27].

In E. coli, the gene cluster for a periplasmic aldehyde oxidoreductase consists of pao $A B C D$ (Figure 3). The paoABC genes code for the three subunits of the trimeric aldehyde oxidoreductase, with PaoA binding two distinct [2Fe2S] clusters, $\mathrm{PaoB}$ binding $\mathrm{FAD}$ and $\mathrm{PaoC}$ containing $\mathrm{MCD}$
[20]. PaoD is predicted to be involved in MCD binding, maturation, and insertion into $\mathrm{PaoC}$, since expression of paoABC in the absence of the $p a o D$ gene leads to inactive and instable PaoABC protein devoid of MCD [20]. Two additional open reading frames have been identified in E. coli, 
coding for XDH homologues, $x d h A B C$ and $x d h D[19,30$, 31 . While both are predicted to have $\mathrm{XDH}$ activities, an $\mathrm{XdhC}$ homologue is not cotranscribed with these genes (Figure 3). However, a second XdhC homologue has been identified in $E$. coli, designated YqeB, which is organized in a single transcriptional unit in the genome. It remains to be elucidated whether YqeB is the XdhC-like chaperone shared by E. coli XdhD and XdhABC.

Three genes, $\operatorname{coxL}$, coxM, and coxS (for large, medium, and small subunits) encode the polypeptides for the CODH enzyme in O. carboxidovorans OM5 (Figure 3) [27]. Two heterotrimers, each composed of one CoxL, CoxM, and CoxS subunit, combine to form a functional aerobic $\mathrm{CODH}$ enzyme. The large subunit contains Moco, the medium subunit binds FAD, and the small subunit has two [2Fe2S] clusters [11]. In addition to these three genes, a number of other accessory genes have also been identified (CoxB, CoxC, CoxH, CoxD, CoxE, CoxF, CoxG, CoxI, and CoxK) that are believed to be required in the processes of regulation, posttranslational modification, and anchorage of the $\mathrm{CODH}$ complex to the cytoplasmic membrane. A number of these accessory genes are membrane-bound proteins themselves (CoxB, CoxC, CoxH, and CoxK), containing several transmembrane helices, and indeed, in O. carboxidovorans OM5, the CODH enzyme itself has been observed to be associated with the inner cytoplasmic membrane [27]. By sequence analysis, several promoters were annotated within the gene region containing 12 genes essential for carboxidotrophic utilization (Figure 3) [27]. CoxD was shown to be a MoxRlike AAA+ ATPase with a predicted function in the stepwise introduction of sulfur and copper in the $\left[\mathrm{CuSMoO}_{2}\right]$ center of the enzyme [32]. The role of CoxF and CoxI was not characterized yet, but both proteins share amino acid homologies to R. capsulatus XdhC (Figure 4).

In contrast, the organization of the B. subtilis puc operon differs as the gene for the $\mathrm{XdhC}$ homologue, $p u c A$, is located upstream of the structural genes pucCDE encoding for an $\mathrm{XDH}$-like protein (Figure 3) [33]. Downstream of pucA, the $p u c B$ gene is located which encodes for a homologue to the CTP: molybdopterin cytidylyltransferase MocA involved in MCD biosynthesis (addition of the CMP moiety to Mo-MPT, [34]) (Figure 1). A knockout of pucA and pucB in B. subtilis decreased growth on hypoxanthine or guanosine as nitrogen source without affecting growth on ammonia or uric acid [33]. PucA has not been purified or characterized on the molecular level so far.

In Arthrobacter nitroguajacolicus, a linear catabolic plasmid containing a total of 103 open reading frames was shown to be responsible for quinaldine degradation [35]. The plasmid contains the operon qoxMLS encoding for the MCD-containing quinaldine dehydrogenase (QoxMbinding MCD, QoxL-binding FAD, and QoxS-binding 2 [2Fe2S] cluster). An XdhC homologue is located immediately downstream of QoxMLS [35]. The qoxMLS genes for quinaldine dehydrogenase were cloned into an expression vector and introduced into $P$. putida KT2440, resulting in the production of an active quinaldine dehydrogenase [35]. This showed that the P. putida XdhC homologue was functionally capable to insert the MCD cofactor into A. nitroguajacolicus
QoxM. Neither the P. putida nor the A. nitroguajacolicus $\mathrm{XdhC}$ homologues were characterized on the molecular level so far.

Figure 4 shows an amino acid sequence alignment of the XdhC-like proteins encoded by these operon structures. From the amino acid sequence alignment, it becomes clear that the overall sequence identity of the XdhC-like proteins from different bacteria is not high $(\sim 15 \%-30 \%)$. There are only very few highly conserved amino acids which are present in all XdhC-like proteins. However, one cysteine residue is conserved through almost all XdhC-like proteins present in the database, which corresponds to cysteine 82 in $R$. capsulatus XdhC (highlighted in red in Figure 4).

\section{A Phylogenetic Point of View of the XdhC Family}

The XdhC family of proteins contains hundreds of members that are bacterial and archaeal proteins, but no homologous sequences are present in eukaryotic genomes. While several organisms such as $R$. capsulatus contain only a single XdhC homologue, a large number of organisms like Rhodobacter sphaeroides or E. coli contain two or more homologues to $\mathrm{XdhC}$. XdhC homologues are also present on strain-specific megaplasmids required for metabolic pathways like the one identified for quinaldine degradation in A. nitroguajacolicus Rü61a [35]. As discussed above, $x d h C$ genes can be present in operons structures in conjunction with their respective structural genes encoding a member of the xanthine oxidase family (Figure 5, marked in bold). In some organisms, a gene organization was identified where genes encoding for XdhC homologues are cotranscribed with genes essential for Moco biosynthesis (Figure 5, underlined).

As obvious from the phylogenetic tree, $\mathrm{XdhC}$ homologues being part of XOR operons in different Proteoand Actinobacteria are clustered. These include XdhC of $R$. capsulatus $\mathrm{XDH}$ and $P$. aeruginosa $\mathrm{XDH}$, which both contain the Mo-MPT form of Moco. In contrast, PucA, the chaperone of B. subtilis XDH [33], forms a cluster with distinct XdhC homologues in different Bacillus strains. Sequence alignments and phylogenetic analysis of the Mococontaining subunits indicate that these $\mathrm{XDH}$ homologues likely contain the MCD form of Moco. Thus, Mo-MPT and $\mathrm{MCD}$-containing XDHs and their respective chaperones might have originated from a common ancestor but have evolved separately during evolution.

The operon organization of the Moco chaperone and the molybdoenzyme partner, for example, like in $R$. capsulatus $x d h A B C[19,22]$ or E. coli paoABCD [20], might indicate that XdhC and PaoD are the system-specific molecular chaperones for R. capsulatus XDH and E. coli AOR, respectively. Other XdhC homologues, including E. coli YqeB, which are not organized in an operon structure with a specific molybdoenzyme, might represent examples for Moco-inserting chaperones responsible for several molybdoenzyme partners. Examples include the flavobacterium Gramella forsetii where an XdhC homologue is a part of an operon containing two putative molybdoenzymes and several putative Moco 


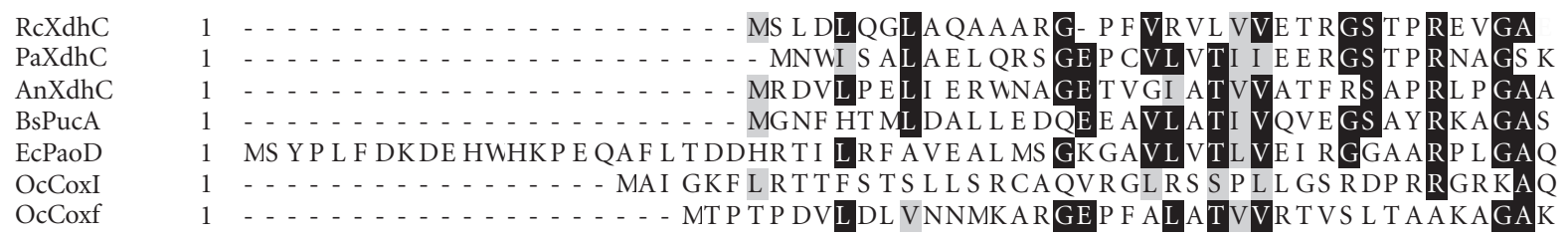

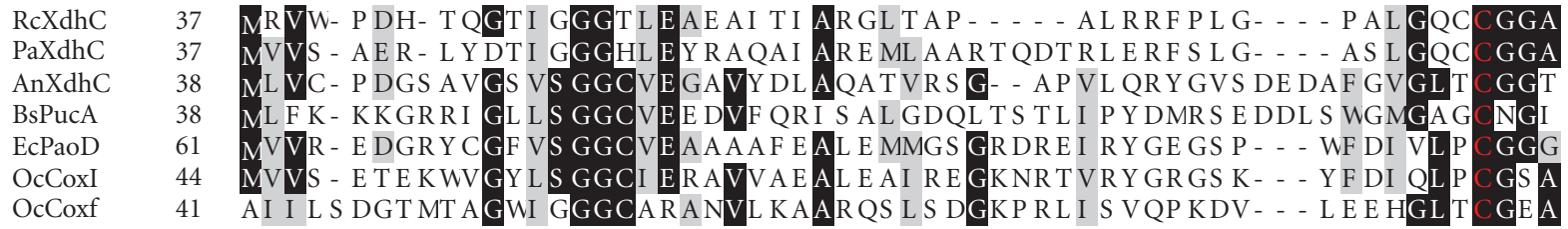

RcXdhC

PaXdhC

AnXdhC

BsPucA

EcPaoD

OcCoxI

OcCoxf

VTLAF EP L DAES L TR I - . . - . . . . . AGP F HARP L T GP DMP L AVQRAL S L ARNS G TVLL F E P MG-

L DI Y I EAMS QATFPEL GDI AADAEAGRPVAL ACI VDHP E AS HI GRKI VVRP QS VE GDL GS I HVHAERI TQEKRRHY - . . . . . . . . . . E KVRDCL HS GKAVTS VI KI E S S HYL F L T

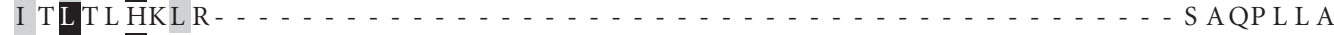

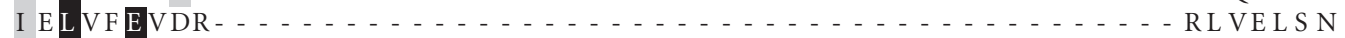
REGVLYANN . . . . . . . . . . . . . . . . . . . . . . . . . . . . . .

RcXdhC 131 QRP P L L L DGWL I ERL AP P AQE L WI WG- - - - AGHVGRAL VA T L APL PHWS I R WADF DE S R

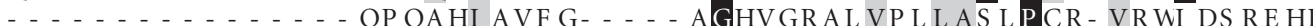
AnXdhC 155 RNI NT TVRRDVL GYL AQGKNGI I AY GP E GQP HGDT I R VF VTS F APAPR MI VF GAI DF AAA BsPucA 141 E NGHF GNWP DA P L QDI QR TVS T L HL P . - . HF DQT T NMF I QR I E P KP RL I L F GAGP DNVP EcPaoD 133 VL NRL E QR KP VGL RY DP QA QS L VCL P - T QT RTGWNL NGF E VGF RP CVRL MI Y GRS L E AQA OcCoxI $\quad 116$ I DELLRS RRPAS MTI TVS HNDGGHDE F ERCYYLPRRQL L A GVGPS AVQL ARLARVS GF D

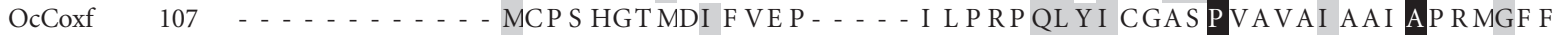

\begin{tabular}{|c|c|c|}
\hline RcXdhC & 186 & $\ldots \ldots$. . . . . . E P I PE TVMP VI AE NP ADLVP L AAAS . . . \\
\hline PaXdhC & 138 & $\ldots \ldots$. . . . . . . A L L P DGVE KVVNDE VL DEVERMP P G . . . \\
\hline AnXdhC & 215 & VARAGS F L GYHVT I CDARP VF T T S S RF P DADE VI VDWP HRYL KS E VE AGR I DD \\
\hline BsPucA & 197 & L ANL A ADT GF S VI VT DWR P AY CT S S L F P KADQL I T AF P E QML S E F QF F P $\ldots$ \\
\hline EcPaoD & 192 & 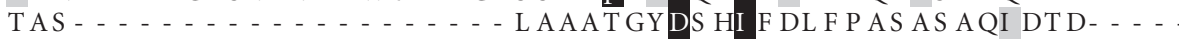 \\
\hline OcCoxI & 176 & $\ldots \ldots$. . . . P DKP TL QAAE L Y DVRI T GVTS P TVL P P L CADS . . . \\
\hline OcCoxf & 150 & $\ldots \ldots$. . . . . . . . VCAPKADHTLF GDT DRL I DGYE I P ADS G- \\
\hline cXdhC & 219 & TY S HAL DLE L CHR I LR - HGF A A CGL I GSQT K WS RF QRRLR DL GHAHA QI S RI A \\
\hline $\mathrm{aXdhC}$ & 171 & THNHP L DLE L T A A I L A R NDF AYF GL I GS KT KR VKF E HR LRE RGVDAE RL QRMR \\
\hline AnXdhC & 275 & THDP KF DI P L LE VALKL P A VGYVGAL GS \\
\hline BsPucA & 253 & THHY QHDQT I I NF L F S - QNL HYI GL L GS \\
\hline EcPaoD & 227 & THT L R L QKLHE LGWS RE E T T QI R \\
\hline OcCoxI & 220 & F HDHNWE LS F LP E I LK- TE AF YI GAL GS RAT HR QRL VQLS RL G I DE MQL KRI H \\
\hline OcCoxf & 187 & S TQGR GDT A ALKS ALS - TP S VYVÄF VGS RKKAS VL RE ELTVAGI AP S L L E TL H \\
\hline RcXdhC & 278 & I S VAAAL L RE RVGHA GL \\
\hline $\mathrm{PaXdhC}$ & 231 & R KQGP S S VTRL TPES RRAHE S - - - \\
\hline AnXdhC & 335 & ETAI S I VAEVI ANKWNGS GARLS ATE L P I HRS HQA GS DMS E VKL Q- \\
\hline BsPucA & 305 & $\ldots \ldots \ldots \ldots-\ldots$ \\
\hline EcF & 286 & 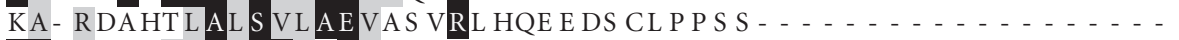 \\
\hline & 279 & S DVALS I L SEI S QL EQDE QQR AL RAF T T DNVHL I QAL AGS P S VNGV \\
\hline OcC & 246 & S I VAEMVE I RR HGQR \\
\hline
\end{tabular}

FIGURE 4: Amino acid sequence alignment of XdhC-like proteins from different bacteria. Shown is an amino acid sequence alignment of the $\mathrm{XdhC}$-like proteins organized in operon structures (from Figure 1) from R. capsulatus (RcXdhC), E. coli (EcPaoD), P. aeruginosa (PaXdhC), O. carboxidovorans (OcCoxF, OcCoxI), A. nitroguajacolicus (AnXdhC), and B. subtilis (BsPucA). A highly conserved cysteine residue present in all XdhC-like proteins is highlighted in red. Identical amino acids are boxed in black, and homologous amino acids are shaded in grey. The amino acid sequence alignment was performed using ClustalW and visualized using Boxshade. 


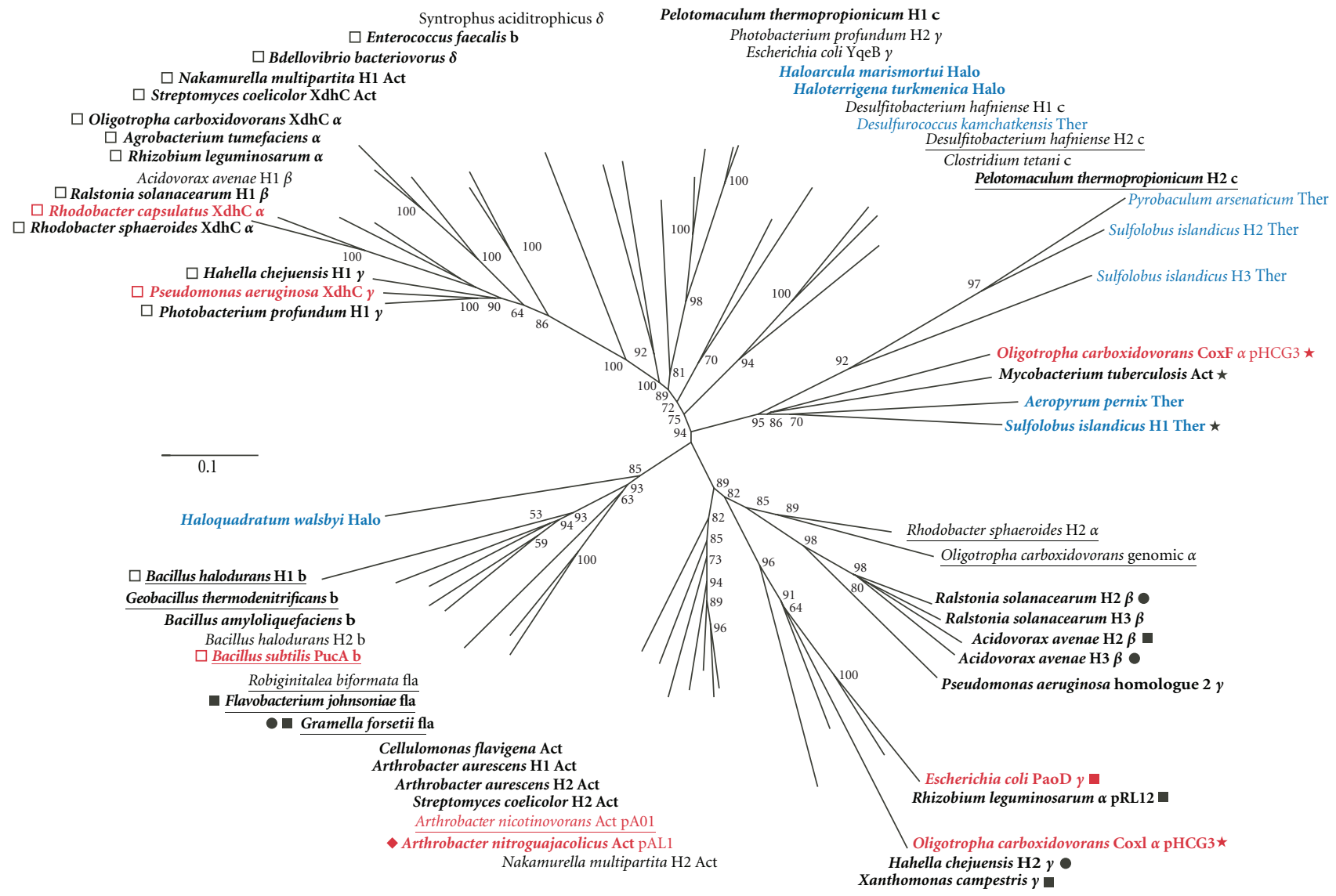

FIgure 5: Phylogenetic tree of XdhC homologues. Protein phylogeny of XdhC homologues based on a full length sequence alignment. The tree was constructed by the neighbor-joining method from a matrix of estimated numbers of amino acid substitutions per site calculated with the Dayhoff option of Phylip. Numbers near branches indicate the bootstrap proportion for 100 replicas using the same method. The scale bar indicates 0.1 substitutions per site. Bacterial XdhC homologues from (partially) characterized operons are marked in red, XdhC homologues from Archaea are marked in blue, and in case several XdhC homologues present in one genome, they are marked by H1, H2, H3; class: b: Bacilli; c: Clostridia; $\alpha$ : Alphaproteobacteria; $\beta$ : Betaproteobacteria; $\gamma$ : Gammaproteobacteria; $\delta$ : Deltaproteobacteria; Act: Actinobacteria; fla: Flavobacteria; Halo: Halobacteria; Ther: Thermoprotei. Underlined XdhC homologues are part of an operon encoding Moco biosynthesis proteins, XdhC homologues in bold are part of an operon encoding a molybdoenzyme, putative molybdoenzyme partners were annotated based on a full length sequence alignment of the Moco binding subunit and by comparison of active site amino acids: PaoC homologues (filled square), XOR homologues (open square), CoxL/CutL homologues (filled star), isoquinoline 1-oxidoreductase homologues (filled circle) and quinaldine 4-oxidase (filled diamond).

biosynthesis proteins. A similar organization is present on the megaplasmid pA01 of Arthrobacter nicotinovorans which includes genes for nicotine dehydrogenase and the ketone dehydrogenase [36]. This megaplasmid contains a single $x d h C$ gene which is cotranscribed with a mocA gene [37]. Since detailed analyses of the role of these $\mathrm{XdhC}$ homologues for several molybdoenzymes have not been analyzed in detail yet, a shared role of these chaperones for several molybdoenzymes remains speculative.

A more complex organization is present in O. carboxidovorans. The cox gene cluster of $O$. carboxidovorans megaplasmid pHCG3 encodes for two XdhC homologues, $\operatorname{cox} F$ and $\operatorname{coxI}$ (Figure 3) [27]. While the exact role of these homologues remains to be elucidated [38], phylogenetic analysis shows that CoxF is more similar to $\mathrm{XdhC}$ homologues found in other putative CODH operons and CoxI shows the highest similarity to E. coli PaoD. Moreover, the genome of $O$. carboxidovorans contains two additional genomic homologues of $\mathrm{XdhC}$, one cotranscribed with the genes for a putative $\mathrm{XDH}$ and the other located in an operon with a MocA homologue.

\section{The Absence of XdhC Affects the Maturation of the Molybdopartner}

To date, a detailed characterization has only been performed for $R$. capsulatus XdhC $[22,28]$. To analyze the function of $\mathrm{XdhC}$ for $\mathrm{XDH}$ in $R$. capsulatus, inactive $\mathrm{XDH}$ was purified from an $R$. capsulatus $x d h C$ mutant strain $[22$, 28]. Analysis of the molybdenum cofactor content of this enzyme demonstrated that in the absence of $\mathrm{XdhC}$, no MoMPT cofactor was present in the XdhAB heterotetramer. In contrast, absorption spectra of inactive $\mathrm{XDH}$ isolated from the $x d h C$ mutant revealed the presence of iron-sulfur clusters 
and flavin adenine dinucleotide, demonstrating that XdhC is not required for the insertion of these cofactors. In the absence of Mo-MPT, XdhC remained associated with the inactive XDH heterodimer devoid of Mo-MPT [22, 28]. In addition, a heterologous system for the expression of $R$. capsulatus XDH in E. coli was established [39]. Here, the $R$. capsulatus $x d h A B C$ genes were coexpressed in $E$. coli, which resulted in a production of a $100 \%$ active XDH containing a full complement of the equatorial $\mathrm{Mo}=\mathrm{S}$ ligand [40]. In the absence of XdhC, Mo-MPT was inserted into XDH in E. coli; however, the content of the equatorial sulfur ligand of molybdenum was drastically reduced [28]. This implied that XdhC is involved in the maturation of Mo-MPT by the addition of the equatorial sulfur ligand to the cofactor prior to the insertion into the XDH apoenzyme (in case of $R$. capsulatus).

The E. coli strain used in this study contained a deletion of the mobAB genes involved in bis-MGD formation [41], which resulted in an accumulation of Mo-MPT to unphysiological high concentrations. Thus, it is believed that MoMPT is unspecifically inserted into $R$. capsulatus $\mathrm{XDH}$ in E. coli during expression likely due to the accumulation of Mo-MPT. Whether one of the E. coli XdhC-like proteins is capable of inserting Mo-MPT into $R$. capsulatus $\mathrm{XDH}$ has not been investigated so far. In E. coli, the level of Moco sulfuration drastically depended on the oxygen concentration, since the amount of sulfurated Moco in XDH largely decreased with a high aeration of the expression cultures [28]. This implied a protective role of XdhC during Moco sulfuration. Similar results were also reported for $C$. acidovorans $\mathrm{XDH}$ expressed in E. coli [29].

For the characterization of $\mathrm{XdhC}$, the protein was purified after heterologous expression in E. coli [28]. Characterization of the purified protein showed that $\mathrm{XdhC}$ is a dimer in solution and the purified protein is able to bind stoichiometric amounts of Mo-MPT or MPT, with dissociation constants of $3.6 \pm 0.1 \mu \mathrm{M}$ and $3.5 \pm 0.3 \mu \mathrm{M}$, respectively [28]. It was also shown that XdhC-bound MoMPT can be inserted into Moco-free apo-XDH. Analysis of protein-protein interactions showed that XdhC specifically interacts with the $\mathrm{XdhB}$ subunit of $\mathrm{XDH}$ for Mo-MPT insertion [28]. The tight interaction of $\mathrm{XdhC}$ with $\mathrm{XdhB}$ underlined the specific role of XdhC in $\mathrm{XDH}$ maturation. For analysis of the role of the highly conserved cysteine residue Cys82 in R. capsulatus XdhC, site-directed mutagenesis was performed to exchange Cys 82 by alanine. Unfortunately, all attempts to purify the $\mathrm{XdhC}-\mathrm{C} 82 \mathrm{~A}$ variant failed, since this protein variant was highly unstable and precipitated completely during purification (unpublished results).

\section{Formation of Sulfurated Moco}

The investigations showed that XdhC binds Mo-MPT, which is produced by MoeA/MogA in the cell $[42,43]$ and protects it from oxidation until the terminal sulfur ligand is inserted. So far, proteins with a specific Moco sulfurase activity were identified in a number of eukaryotes. The first report was given by Wahl et al. [44] describing a mutation in the Drosophila melanogaster maroon-like locus (ma-l) which impaired the activity of $\mathrm{XDH}$ and $\mathrm{AO}$, while the activity of sulfite oxidase remained unaffected [45]. Eukaryotic Moco sulfurase are two-domain proteins with an N-terminal domain showing homologies to bacterial Lcysteine desulfurase (E.C. 2.8.1.7) of the NifS family and a C-terminal domain with Moco-binding properties [46, 47], however, without homologies to the bacterial XdhC family. In general, L-cysteine desulfurase are homodimeric proteins that utilize pyridoxal 5-phosphate (PLP) to catalyze the reductive elimination of sulfur from L-cysteine, resulting in the formation of alanine and an enzyme-bound cysteine persulfide intermediate [48]. R. capsulatus and E. coli contain several L-cysteine desulfurase which could act as a sulfur donor for the sulfuration of Moco in these bacterial systems. The E. coli L-cysteine desulfurase are IscS, CsdA, and SufS [49], and the R. capsulatus homologues were named NifS (specific for nitrogenase), NifS2, NifS3, and NifS4 [50].

A specific role for the three $R$. capsulatus L-cysteine desulfurase NifS2, NifS3, and NifS4 had not been assigned before. Interposon mutagenesis of the nifS2, nifS3, and nifS4 genes showed that nifS2 strains displayed no particular XDH phenotype and that nifS3 and nifS4 are apparently essential for the viability of $R$. capsulatus [50]. To identify the specific L-cysteine desulfurase for the sulfuration of Moco in $R$. capsulatus, a direct E. coli two-hybrid screen was applied and the detected interaction partners were verified by surface plasmon resonance (SPR) measurements using the purified proteins. The L-cysteine desulfurase NifS4 of $R$. capsulatus was identified to specifically interact with $\mathrm{XdhC}$ with a dissociation constant of $0.64 \mu \mathrm{M}$ [50]. It was identified that NifS4 sulfurates Moco while it is bound to XdhC. An interaction of NifS4 with XDH was excluded. Thus, the XdhC-NifS4 pair can be considered as the prokaryotic counterpart to specific Moco sulfatases identified in eukaryotes. However, since interposon mutagenesis of nifS4 was not successful, the corresponding protein appears to have an additional role in another essential sulfur transfer pathway in the cell. NifS4 mobilizes sulfur from L-cysteine by formation of a protein-bound persulfide intermediate and transfers this sulfur further to XdhC-bound Moco. This reaction was shown to be more effective than the chemical sulfuration of Moco using sulfide as sulfur source. Further studies clearly showed that Moco is sulfurated before the insertion into $\mathrm{XDH}$, while it is bound to $\mathrm{XdhC}$.

\section{A Model for the Insertion of Sulfurated Moco and Maturation of Molybdoenzymes}

The assembly of $\mathrm{XDH}$ is a highly ordered process, which involves the synthesis of the XdhA and XdhB subunits, the dimerization of both subunits, the insertion of FeSI, FeSII, and FAD into the XdhA subunit, the dimerization of two $(\alpha \beta)$ dimers via the $\mathrm{XdhB}$ subunit, and finally, the insertion of sulfurated Moco into XdhB, resulting in an active enzyme (Figure 6) [51]. The biosynthesis of Moco is additionally a complex process involving more than a dozen different proteins $[52,53]$, with the insertion of sulfurated Moco by the $\mathrm{XdhC}$ protein being the last step of $\mathrm{XDH}$ maturation [22]. 


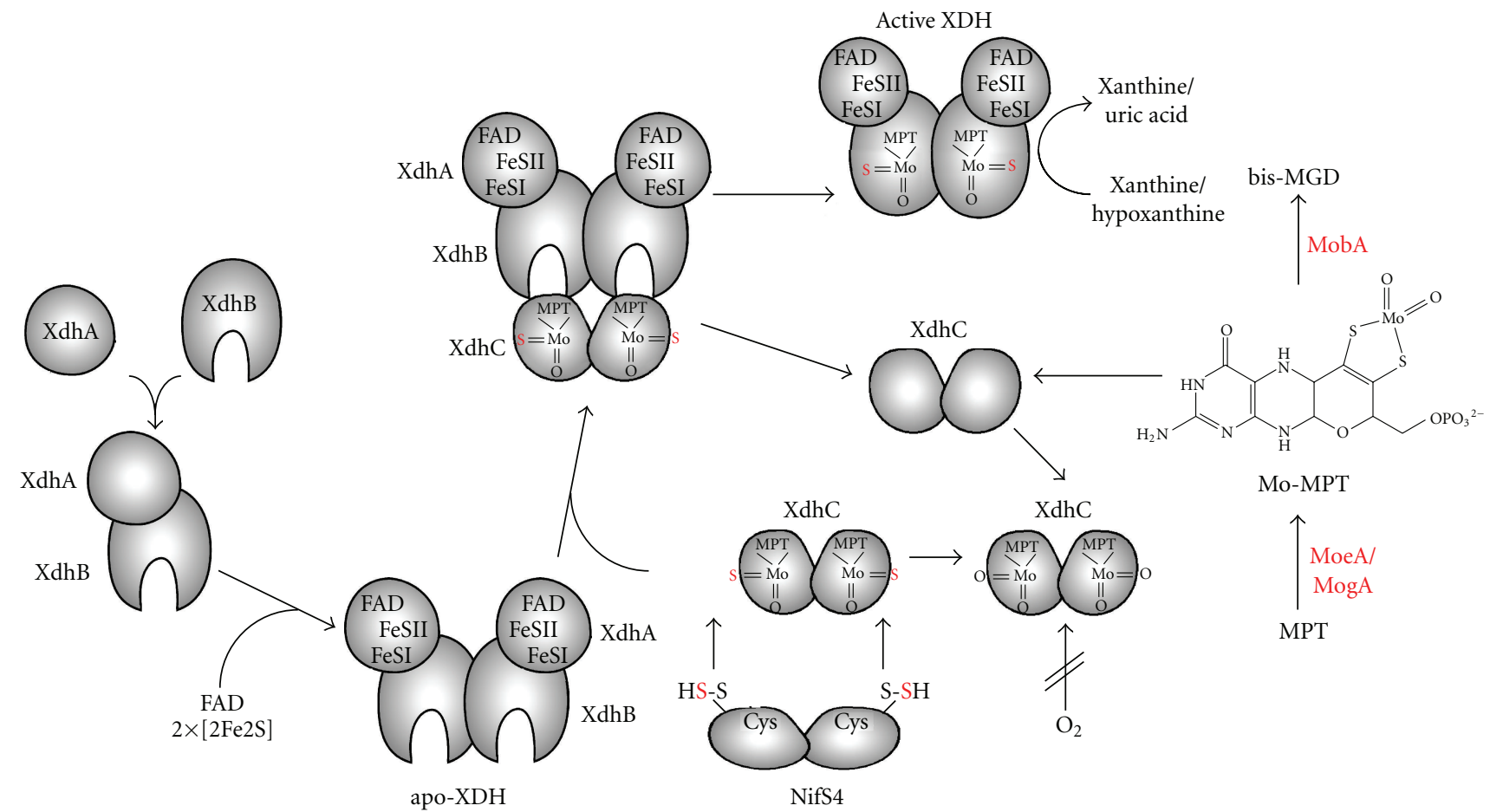

Figure 6: Model for the role of XdhC in the assembly of R. capsulatus XDH. The assembly of R. capsulatus XDH involves the synthesis of the XdhA and XdhB subunits, the dimerization of both subunits, the insertion of FeSI, FeSII, and FAD into the XdhA subunit, dimerization of two $(\alpha \beta)$ dimers via the XdhB subunit to form the Moco-free apo-XDH, and finally, insertion of sulfurated Moco into XdhC. Mo-MPT is produced by molybdenum insertion into MPT catalyzed by MogA and MoeA and can further be converted to bis-MGD by the MobA protein. It is suggested that MoeA/MogA/MobA and $\mathrm{XdhC}$ form a complex in the cell. $\mathrm{XdhC}$ binds Mo-MPT, and the equatorial Mo=S is inserted into Moco before its incorporation into XDH by the sulfurtransferase function of the NifS4 protein. XdhC protects Moco from oxidation and interacts with $\mathrm{XDH}$ for Moco insertion.

Moco is produced from MPT by the MogA-MoeA complex, catalyzing the ATP-dependent ligation of the molybdenum atom to MPT (Figure 6) [54, 55]. Synthesized Mo-MPT can subsequently be transferred either to MobA, converting it into bis-MGD (which is inserted into enzymes of the DMSO reductase family) [56], or to XdhC, forming the sulfurated form of Moco by exchange of an oxoligand with sulfur (which is inserted into $\mathrm{XDH}$ ). $\mathrm{XdhC}$ interacts with MobA and thereby inhibits the transfer of Moco to MobA [43]. Further, it is believed that XdhC dissociates from this complex to interact with the L-cysteine desulfurase NifS4. NifS4 directly interacts with XdhC and exchanges the equatorial oxygen ligand specifically by a sulfur ligand. The sulfur for this reaction originated from L-cysteine and a persulfide group is formed on NifS4 in the course of the reaction. Further, XdhC has to dissociate from NifS4 after the sulfuration reaction to interact with XdhB. The present results for R. capsulatus $\mathrm{XDH}$ demonstrate that dimerization via the $\beta$-subunits is required to stabilize a structure of the protein that makes the protein suitable for Moco insertion [51]. Since XdhC is a dimer itself, it is suggested that the $\mathrm{XdhC}$ dimer interacts with the $\mathrm{XdhB}$ dimer simultaneously inserting matured and sulfurated Mo-MPT into both active sites of XdhB (Figure 6). The insertion of sulfurated Moco into $\mathrm{XDH}$ is strictly regulated in $R$. capsulatus, because in vivo dioxo-Moco is not inserted into $\mathrm{XDH}$ [28]. The mode of control of this step has not been identified to date. After this reaction, $\mathrm{XDH}$ is correctly folded and $\mathrm{XdhC}$ dissociates from the complex. Since XdhC is very labile in the absence of Moco, it is believed that after performing the insertion reaction, $\mathrm{XdhC}$ is degraded in the cell.

Thus, XdhC performs several control reactions: (i) to ensure that Moco is sulfurated by the interaction with the L-cysteine desulfurase NifS4 [50] before insertion into XDH and (ii) to insert the sulfurated Moco in the formed $(\alpha \beta)_{2}$ heterotetramer of XDH. Because Moco is deeply buried in the protein, it is also believed that $\mathrm{XdhC}$ acts as a chaperone being involved in proper folding of XDH after Moco insertion [22]. The model shows that apo-XDH exists in a Moco competent "open" conformation until the insertion of sulfurated Moco and that after the insertion reaction, the protein adapts the final active "closed" conformation, which is incapable to accept Moco (Figure 6).

\section{Functional XdhC Homologues in Eukaryotes}

Eukaryotic Moco sulfurase are two-domain proteins containing one protein domain with homologies to L-cysteine desulfurase and a second protein domain belonging to a superfamily of $\beta$-strand-rich domains, named MOSC domains [57]. These MOSC domain proteins are characterized to contain an absolutely conserved cysteine residue. The 
MOSC domain was predicted to be a sulfur-carrier domain that receives the sulfur abstracted by the PLP-dependent L-cysteine desulfurase domain on its cysteine residue and delivers it for the formation of, for example, sulfurated Moco in case of the Moco sulfurase [57]. Bacterial homologues to the MOSC superfamily were also identified, like the $E$. coli YiiM protein [58], and one to four orthologs of YiiM per genome are present in diverse bacteria such as $\alpha$ - and $\gamma$-proteobacteria and gram-positive bacteria [57]. The E. coli YiiM protein was characterized to be involved in the detoxification of 6-N-hydroxylaminopurine to adenine, a pathway which depends on the presence of active Moco [58]. It could be clearly shown that the role of YiiM is distinct from the formation of sulfurated Moco for enzymes of the xanthine oxidase family [58]. However, the precise role and the involvement of Moco in the reaction remains to be elucidated for YiiM.

The currently best characterized eukaryotic Moco sulfurase is ABA3 from Arabidopsis thaliana [59, 60]. ABA3 is composed of an N-terminal NifS-like domain and a C-terminal MOSC domain. L-cysteine desulfurase activity and sulfur transferase activity of ABA3 were shown to be essential for the activity of AO, being involved in abscisic acid (ABA) biosynthesis in plants. It has been suggested that a persulfide sulfur is transferred from the NifS-like domain of $\mathrm{ABA} 3$ to the Moco in $\mathrm{AO}$ of plants [60]. More recent studies showed that the C-terminal MOSC domain is able to bind Moco in a $1: 1$ ratio with a dissociation constant of $0.55 \pm 0.14 \mu \mathrm{M}$, implying that the C-terminus of ABA3 represents the functional homologue of $\mathrm{XdhC}$ in bacteria [61]. In addition, it was shown that the Moco bound on ABA3 existed in its sulfurated form. However, in contrast to the case in bacteria, Wollers et al. [61] proposed that the role of the ABA3 C-terminus in activation of $\mathrm{XDH}$ and $\mathrm{AO}$ occurs posttranslationally, after the insertion of Mo-MPT in these enzymes. Evidence for this assumption was obtained from experiments showing that the activity of these enzymes in ABA3 C-terminus mutant extracts (sir33) was increased by a chemical, nonenzymatical sulfuration procedure. This implied that $\mathrm{AO}$ in plants is present in both a sulfo and a desulfo form, which can posttranslationally be activated by $\mathrm{ABA} 3$. Thus, in plants, $\mathrm{AO}$ and $\mathrm{XDH}$ remain inactive until one of the two oxygen ligands is replaced by a sulfur atom delivered by ABA3. By this mechanism, it was suggested that the plant is able to rapidly increase the activities of $\mathrm{AO}$ and $\mathrm{XDH}$ without de novo synthesis of the apoproteins, representing a mechanism of rapid posttranslational regulation $[59,61]$. Unfortunately, the authors did not consider the possibility that in the sir3-3 $A B A 3$ mutant, the nonsulfurated Moco was inserted unspecifically into AO. Following the model by Bittner's group, it remains to be elucidated how $\mathrm{AO}$ and $\mathrm{XDH}$ receive their sulfurated Moco. In plant $\mathrm{AO}$ and $\mathrm{XDH}$, this could occur either by an exchange of a sulfurated with a nonsulfurated Moco by aid of ABA3 or by exchange of solely the sulfide-ligand. Both models are largely different from the system in bacteria, where a quality control mechanism guarantees that only sulfurated Moco is inserted into enzymes of the xanthine oxidase family, and a posttranslational regulation of enzyme activity via its sulfuration level does not occur. In bacteria, after insertion of sulfurated Moco which is deeply buried into the enzyme, the enzyme is correctly folded. Thus, eukaryotic Moco sulfurase must have developed a system for the unfolding of the inactive holoenzyme to either extract the Moco from it or to sulfurate the Moco while bound to the target enzyme. This shows that for a similar function, the eukaryotic and the prokaryotic systems must have evolved independently.

\section{Concluding Remarks}

This paper highlights the key roles of the bacterial XdhC family of system-specific chaperones in Moco modification and insertion into enzymes of the xanthine oxidase family. Due to a lack of information on other XdhC-like proteins, this paper is mainly focussed on the role of $R$. capsulatus $\mathrm{XdhC}$ for XDH. However, on the basis of sequence similarities and gene organizations in other bacteria, we believe that $R$. capsulatus XdhC represents an example of a large family of XdhC-like proteins which perform the same function for their specific molybdoenzyme partners. These specific molecular XdhC-like chaperones are involved in the late steps of Moco biosynthesis by protecting Mo-MPT against oxidation for its final maturation step, the exchange of the equatorial oxygen ligand at the Mo center against a sulfide group. All XdhC-like proteins contain a highly conserved cysteine residue, which might perform a similar function as the conserved cysteine in the MOSC-like domains of eukaryotic Moco sulfatases. So far, the role of this cysteine residue is not completely clear in both systems. It could either act as a sulfur-transferring cysteine on which a persulfide is formed during the reaction, but it also could serve as a ligand to the protein-bound Mo-MPT, thus, either stabilizing the bound Moco or representing a ligand to generate an electron environment that facilitates the sulfur/oxygen exchange reaction. After synthesis of the mono-oxo Moco, the cofactor is specifically inserted into a target enzyme. Conclusively, the XdhC family of proteins must specifically recognize and interact with its target protein partner. During the maturation step of the apomolybdoenzyme, XdhC must stabilize a competent state of the apoenzyme for cofactor insertion. The matured Moco is inserted into the catalytic site of the enzyme which, in its final form, is deeply buried in the enzyme. After final folding of the molybdoenzyme, $\mathrm{XdhC}$ has performed its role and is no longer required for enzyme activity and consequently dissociates from the holomolybdoenzyme. In total, these XdhC proteins perform versatile roles for Moco and molybdoenzyme maturation, involving the interaction with different proteins, which has to be highly specific. Attempts to solve the crystal structure of XdhC are under investigation, which will shed light into the interaction sites and Moco binding site of this protein. In eukaryotes, functional homologues to XdhC exist, which have further evolved from this system and are fused to one interaction partner, the L-cysteine desulfurase which forms sulfurated Mo-MPT. Further investigations will clarify whether the C-terminal MOSC domain of eukaryotic Moco sulfurase performs a similar role like XdhC in bacteria. In 
total, the XdhC family represents an exquisite model to study the maturation of molybdoenzymes.

\section{Acknowledgments}

This work was supported by continuous Deutsche Forschungsgemeinschaft grants, by the DAAD PROCOPE Program, and the Cluster of Excellence "Unifying Concepts in Catalysis" coordinated by the Technische Universität Berlin. Authors thank K. V. Rajagopalan (Duke University) for his constant support.

\section{References}

[1] R. Hille, “The mononuclear molybdenum enzymes," Chemical Reviews, vol. 96, no. 7, pp. 2757-2816, 1996.

[2] K. V. Rajagopalan and J. L. Johnson, "The pterin molybdenum cofactors," Journal of Biological Chemistry, vol. 267, no. 15, pp. 10199-10202, 1992.

[3] G. Schwarz, "Molybdenum cofactor biosynthesis and deficiency," Cellular and Molecular Life Sciences, vol. 62, no. 23, pp. 2792-2810, 2005.

[4] R. Hille, "Molybdenum-containing hydroxylases," Archives of Biochemistry and Biophysics, vol. 433, no. 1, pp. 107-116, 2005.

[5] M. J. Romão, M. Archer, I. Moura et al., "Crystal structure of the xanthine oxidase-related aldehyde oxido-reductase from D. gigas," Science, vol. 270, no. 5239, pp. 1170-1176, 1995.

[6] J. Rebelo, S. Macieira, J. M. Dias et al., "Gene sequence and crystal structure of the aldehyde oxidoreductase from Desulfovibrio desulfuricans ATCC 27774," Journal of Molecular Biology, vol. 297, no. 1, pp. 135-146, 2000.

[7] C. Enroth, B. T. Eger, K. Okamoto, T. Nishino, T. Nishino, and E. F. Pai, "Crystal structures of bovinemilk xanthine dehydrogenase and xanthine oxidase: structure-based mechanism of conversion," Proceedings of the National Academy of Sciences of the United States of America, vol. 97, no. 20, pp. 10723-10728, 2000.

[8] J. J. Truglio, K. Theis, S. Leimkühler, R. Rappa, K. V. Rajagopalan, and C. Kisker, "Crystal structures of the active and alloxanthine-inhibited forms of xanthine dehydrogenase from Rhodobacter capsulatus," Structure, vol. 10, no. 1, pp. 115-125, 2002.

[9] I. Bonin, B. M. Martins, V. Purvanov, S. Fetzner, R. Huber, and H. Dobbek, "Active site geometry and substrate recognition of the molybdenum hydroxylase quinoline 2-oxidoreductase," Structure, vol. 12, no. 8, pp. 1425-1435, 2004.

[10] M. Unciuleac, E. Warkentin, C. C. Page, M. Boll, and U. Ermler, "Structure of a xanthine oxidase-related 4hydroxybenzoyl-CoA reductase with an additional [4Fe-4S] cluster and an inverted electron flow," Structure, vol. 12, no. 12, pp. 2249-2256, 2004.

[11] H. Dobbek, L. Gremer, O. Meyer, and R. Huber, "Crystal structure and mechanism of CO dehydrogenase, a molybdo ironsulfur flavoprotein containing S-selanylcysteine," Proceedings of the National Academy of Sciences of the United States of America, vol. 96, no. 16, pp. 8884-8889, 1999.

[12] P. Hänzelmann, H. Dobbek, L. Gremer, R. Huber, and O. Meyer, "The effect of intracellular molybdenum in Hydrogenophaga pseudoflava on the crystallographic structure of the seleno-molybdo-iron-sulfur flavoenzyme carbon monoxide dehydrogenase," Journal of Molecular Biology, vol. 301, no. 5, pp. 1221-1235, 2000.
[13] C. D. Brondino, M. J. Romão, I. Moura, and J. J. G. Moura, "Molybdenum and tungsten enzymes: the xanthine oxidase family," Current Opinion in Chemical Biology, vol. 10, no. 2, pp. 109-114, 2006.

[14] E. Garattini, R. Mendel, M. J. Romão, R. Wright, and M. Terao, "Mammalian molybdo-flavoenzymes, an expanding family of proteins: structure, genetics, regulation, function and pathophysiology," Biochemical Journal, vol. 372, no. 1, pp. 15-32, 2003.

[15] H. Dobbek, L. Gremer, R. Kiefersauer, R. Huber, and O. Meyer, "Catalysis at a dinuclear $[\mathrm{CuSMo}(=\mathrm{O}) \mathrm{OH}]$ cluster in a $\mathrm{CO}$ dehydrogenase resolved at 1.1-Å resolution," Proceedings of the National Academy of Sciences of the United States of America, vol. 99, no. 25, pp. 15971-15976, 2002.

[16] E. Garattini, M. Fratelli, and M. Terao, "Mammalian aldehyde oxidases: genetics, evolution and biochemistry," Cellular and Molecular Life Sciences, vol. 65, no. 7-8, pp. 1019-1048, 2008.

[17] T. Nishino, "The conversion of xanthine dehydrogenase to xanthine oxidase and the role of the enzyme in reperfusion injury," Journal of Biochemistry, vol. 116, no. 1, pp. 1-6, 1994.

[18] T. Nishino, K. Okamoto, Y. Kawaguchi et al., "Mechanism of the conversion of xanthine dehydrogenase to xanthine oxidase: identification of the two cysteine disulfide bonds and crystal structure of a non-convertible rat liver xanthine dehydrogenase mutant," Journal of Biological Chemistry, vol. 280, no. 26, pp. 24888-24894, 2005.

[19] S. Leimkühler, M. Kern, P. S. Solomon et al., "Xanthine dehydrogenase from the phototrophic purple bacterium Rhodobacter capsulatus is more similar to its eukaryotic counterparts than to prokaryotic molybdenum enzymes," Molecular Microbiology, vol. 27, no. 4, pp. 853-869, 1998.

[20] M. Neumann, G. Mittelstädt, C. Iobbi-Nivol et al., "A periplasmic aldehyde oxidoreductase represents the first molybdopterin cytosine dinucleotide cofactor containing molybdoflavoenzyme from Escherichia coli," FEBS Journal, vol. 276, no. 10, pp. 2762-2774, 2009.

[21] C. Kisker, H. Schindelin, and D. C. Rees, "Molybdenumcofactor-containing enzymes: structure and mechanism," Annual Review of Biochemistry, vol. 66, pp. 233-267, 1997.

[22] S. Leimkühler and W. Klipp, "Role of XDHC in molybdenum cofactor insertion into xanthine dehydrogenase of Rhodobacter capsulatus," Journal of Bacteriology, vol. 181, no. 9, pp. 27452751, 1999.

[23] F. Blasco, J.-P. Dos Santos, A. Magalon et al., "NarJ is a specific chaperone required for molybdenum cofactor assembly in nitrate reductase A of Escherichia coli," Molecular Microbiology, vol. 28, no. 3, pp. 435-447, 1998.

[24] M. Ilbert, V. Méjean, M.-T. Giudici-Orticoni, J.-P. Samama, and C. Iobbi-Nivol, "Involvement of a mate chaperone (TorD) in the maturation pathway of molybdoenzyme TorA," Journal of Biological Chemistry, vol. 278, no. 31, pp. 28787-28792, 2003.

[25] S. Tranier, C. Iobbi-Nivol, C. Birck et al., "A novel protein fold and extreme domain swapping in the dimeric TorD chaperone from Shewanella massilia," Structure, vol. 11, no. 2, pp. 165174, 2003.

[26] O. Genest, V. Méjean, and C. Iobbi-Nivol, "Multiple roles of TorD-like chaperones in the biogenesis of molybdoenzymes," FEMS Microbiology Letters, vol. 297, no. 1, pp. 1-9, 2009.

[27] B. Santiago, U. Schübel, C. Egelseer, and O. Meyer, "Sequence analysis, characterization and CO-specific transcription of the cox gene cluster on the megaplasmid pHCG3 of Oligotropha carboxidovorans," Gene, vol. 236, no. 1, pp. 115-124, 1999. 
[28] M. Neumann, M. Schulte, N. Jünemann, W. Stöcklein, and S. Leimkühler, "Rhodobacter capsulatus XdhC is involved in molybdenum cofactor binding and insertion into xanthine dehydrogenase," Journal of Biological Chemistry, vol. 281, no. 23, pp. 15701-15708, 2006.

[29] N. V. Ivanov, F. Hubálek, M. Trani, and D. E. Edmondson, "Factors involved in the assembly of a functional molybdopyranopterin center in recombinant Comamonas acidovorans xanthine dehydrogenase," European Journal of Biochemistry, vol. 270, no. 23, pp. 4744-4754, 2003.

[30] S. G. Kozmin and R. M. Schaaper, "Molybdenum cofactordependent resistance to $\mathrm{N}$-hydroxylated base analogs in Escherichia coli is independent of MobA function," Mutation Research, vol. 619, no. 1-2, pp. 9-15, 2007.

[31] H. Xi, B. L. Schneider, and L. Reitzer, "Purine catabolism in Escherichia coli and function of xanthine dehydrogenase in purine salvage," Journal of Bacteriology, vol. 182, no. 19, pp. 5332-5341, 2000.

[32] A. Pelzmann, M. Ferner, M. Gnida, W. Meyer-Klaucke, and O. Meyer, "The CoxD protein of Oligotropha carboxidovorans is a predicted AAA+ ATPase chaperone involved in the biogenesis of the CO dehydrogenase $\left[\mathrm{CuSMoO}_{2}\right]$ cluster," Journal of Biological Chemistry, vol. 284, no. 14, pp. 9578-9586, 2009.

[33] A. C. Schultz, P. Nygaard, and H. H. Saxild, "Functional analysis of 14 genes that constitute the purine catabolic pathway in Bacillus subtilis and evidence for a novel regulon controlled by the PucR transcription activator," Journal of Bacteriology, vol. 183, no. 11, pp. 3293-3302, 2001.

[34] M. Neumann, G. Mittelstädt, F. Seduk, C. Iobbi-Nivol, and S. Leimkühler, "MocA is a specific cytidylyltransferase involved in molybdopterin cytosine dinucleotide biosynthesis in Escherichia coli," Journal of Biological Chemistry, vol. 284, no. 33, pp. 21891-21898, 2009.

[35] K. Parschat, B. Hauer, R. Kappl, R. Kraft, J. Hüttermann, and S. Fetzner, "Gene cluster of Arthrobacter ilicis Ru61a involved in the degradation of quinaldine to anthranilate. Characterization and functional expression of the quinaldine 4-oxidase qoxLMS genes," Journal of Biological Chemistry, vol. 278, no. 30, pp. 27483-27494, 2003.

[36] C. Menéndez, G. Igloi, H. Henninger, and R. Brandsch, "A pAO1-encoded molybdopterin cofactor gene (moaA) of Arthrobacter nicotinovorans: characterization and site-directed mutagenesis of the encoded protein," Archives of Microbiology, vol. 164, no. 2, pp. 142-151, 1995.

[37] D. Baitsch, C. Sandu, R. Brandsch, and G. L. Igloi, "Gene cluster on pAO1 of Arthrobacter nicotinovorans involved in degradation of the plant alkaloid nicotine: cloning, purification, and characterization of 2,6-dihydroxypyridine 3-hydroxylase," Journal of Bacteriology, vol. 183, no. 18, pp. 5262-5267, 2001.

[38] S. Fuhrmann, M. Ferner, T. Jeffke, A. Henne, G. Gottschalk, and O. Meyer, "Complete nucleotide sequence of the circular megaplasmid pHCG3 of Oligotropha carboxidovorans: function in the chemolithoautotrophic utilization of $\mathrm{CO}, \mathrm{H}_{2}$ and $\mathrm{CO}_{2}$," Gene, vol. 322, no. 1-2, pp. 67-75, 2003.

[39] S. Leimkühler, R. Hodson, G. N. George, and K. V. Rajagopalan, "Recombinant Rhodobacter capsulatus xanthine dehydrogenase, a useful model system for the characterization of protein variants leading to xanthinuria I in humans," Journal of Biological Chemistry, vol. 278, no. 23, pp. 2080220811, 2003.

[40] S. Leimkühler, A. L. Stockert, K. Igarashi, T. Nishino, and R. Hille, "The role of active site glutamate residues in catalysis of Rhodobacter capsulatus xanthine dehydrogenase," Journal of Biological Chemistry, vol. 279, no. 39, pp. 40437-40444, 2004.
[41] T. Palmer, A. Vasishta, P. W. Whitty, and D. H. Boxer, "Isolation of protein FA, a product of the mob locus required for molybdenum cofactor biosynthesis in Escherichia coli," European Journal of Biochemistry, vol. 222, no. 2, pp. 687-692, 1994.

[42] S. Leimkühler, S. Angermüller, G. Schwarz, R. R. Mendel, and W. Klipp, "Activity of the molybdopterin-containing xanthine dehydrogenase of Rhodobacter capsulatus can be restored by high molybdenum concentrations in a moeA mutant defective in molybdenum cofactor biosynthesis," Journal of Bacteriology, vol. 181, no. 19, pp. 5930-5939, 1999.

[43] M. Neumann, W. Stöcklein, and S. Leimkühler, "Transfer of the molybdenum cofactor synthesized by Rhodobacter capsulatus MoeA to XdhC and MobA," Journal of Biological Chemistry, vol. 282, no. 39, pp. 28493-28500, 2007.

[44] R. C. Wahl, C. K. Warner, V. Finnerty, and K. V. Rajagopalan, "Drosophila melanogaster ma-l mutants are defective in the sulfuration of desulfo Mo hydroxylases," Journal of Biological Chemistry, vol. 257, no. 7, pp. 3958-3962, 1982.

[45] V. Finnerty, M. McCarron, and G. B. Johnson, "Gene expression in Drosophila: post-translational modification of aldehyde oxidase and xanthine dehydrogenase," Molecular and General Genetics, vol. 172, no. 1, pp. 37-43, 1979.

[46] L. Amrani, J. Primus, A. Glatigny, L. Arcangeli, C. Scazzocchio, and V. Finnerty, "Comparison of the sequences of the Aspergillus nidulans $h x B$ and Drosophila melanogaster ma-I genes with nifS from Azotobacter vinelandii suggests a mechanism for the insertion of the terminal sulphur atom in the molybdopterin cofactor," Molecular Microbiology, vol. 38, no. 1, pp. 114-125, 2000.

[47] R. R. Mendel and F. Bittner, "Cell biology of molybdenum," Biochimica et Biophysica Acta, vol. 1763, no. 7, pp. 621-635, 2006.

[48] L. Zheng, R. H. White, V. L. Cash, and D. R. Dean, "Mechanism for the desulfurization of L-cysteine catalyzed by the NIFs gene product," Biochemistry, vol. 33, no. 15, pp. 4714-4720, 1994.

[49] T. Kurihara, H. Mihara, S.-I. Kato, T. Yoshimura, and N. Esaki, "Assembly of iron-sulfur clusters mediated by cysteine desulfurases, IscS, CsdB and CSD, from Escherichia coli," Biochimica et Biophysica Acta, vol. 1647, no. 1-2, pp. 303-309, 2003.

[50] M. Neumann, W. Stöcklein, A. Walburger, A. Magalon, and S. Leimkühler, "Identification of a Rhodobacter capsulatus Lcysteine desulfurase that sulfurates the molybdenum cofactor when bound to XdhC and before its insertion into xanthine dehydrogenase," Biochemistry, vol. 46, no. 33, pp. 9586-9595, 2007.

[51] S. Schumann, M. Saggu, N. Möller et al., "The mechanism of assembly and cofactor insertion into Rhodobacter capsulatus xanthine dehydrogenase," Journal of Biological Chemistry, vol. 283, no. 24, pp. 16602-16611, 2008.

[52] K. V. Rajagopalan, "Biosynthesis of the molybdenum cofactor in Escherichia coli and Salmonella," in Cellular and Molecular Biology, F. C. Neidhardt, Ed., pp. 674-679, ASM Press, Washington, DC, USA, 1996.

[53] G. Schwarz, R. R. Mendel, and M. W. Ribbe, "Molybdenum cofactors, enzymes and pathways," Nature, vol. 460, no. 7257, pp. 839-847, 2009.

[54] J. Nichols and K. V. Rajagopalan, "Escherichia coli MoeA and MogA: function in metal incorporation step of molybdenum cofactor biosynthesis," Journal of Biological Chemistry, vol. 277, no. 28, pp. 24995-25000, 2002. 
[55] J. D. Nichols and K. V. Rajagopalan, "In vitro molybdenum ligation to molybdopterin using purified components," Journal of Biological Chemistry, vol. 280, no. 9, pp. 7817-7822, 2005.

[56] T. Palmer, C.-L. Santini, C. Iobbi-Nivol, D. J. Eaves, D. H. Boxer, and G. Giordano, "Involvement of the narJ and mob gene products in distinct steps in the biosynthesis of the molybdoenzyme nitrate reductase in Escherichia coli," Molecular Microbiology, vol. 20, no. 4, pp. 875-884, 1996.

[57] V. Anantharaman and L. Aravind, "MOSC domains: ancient, predicted sulfur-carrier domains, present in diverse metalsulfur cluster biosynthesis proteins including Molybdenum cofactor sulfurases," FEMS Microbiology Letters, vol. 207, no. 1, pp. 55-61, 2002.

[58] S. G. Kozmin, P. Leroy, Y. I. Pavlov, and R. M. Schaaper, "YcbX and yiiM, two novel determinants for resistance of Escherichia coli to N-hydroxylated base analogues," Molecular Microbiology, vol. 68, no. 1, pp. 51-65, 2008.

[59] F. Bittner, M. Oreb, and R. R. Mendel, "ABA3 is a molybdenum cofactor sulfurase required for activation of aldehyde oxidase and xanthine dehydrogenase in Arabidopsis thaliana," Journal of Biological Chemistry, vol. 276, no. 44, pp. 40381-40384, 2001.

[60] T. Heidenreich, S. Wollers, R. R. Mendel, and F. Bittner, "Characterization of the NifS-like domain of ABA3 from Arabidopsis thaliana provides insight into the mechanism of molybdenum cofactor sulfuration," Journal of Biological Chemistry, vol. 280, no. 6, pp. 4213-4218, 2005.

[61] S. Wollers, T. Heidenreich, M. Zarepour et al., "Binding of sulfurated molybdenum cofactor to the C-terminal domain of ABA3 from Arabidopsis thaliana provides insight into the mechanism of molybdenum cofactor sulfuration," Journal of Biological Chemistry, vol. 283, no. 15, pp. 9642-9650, 2008. 

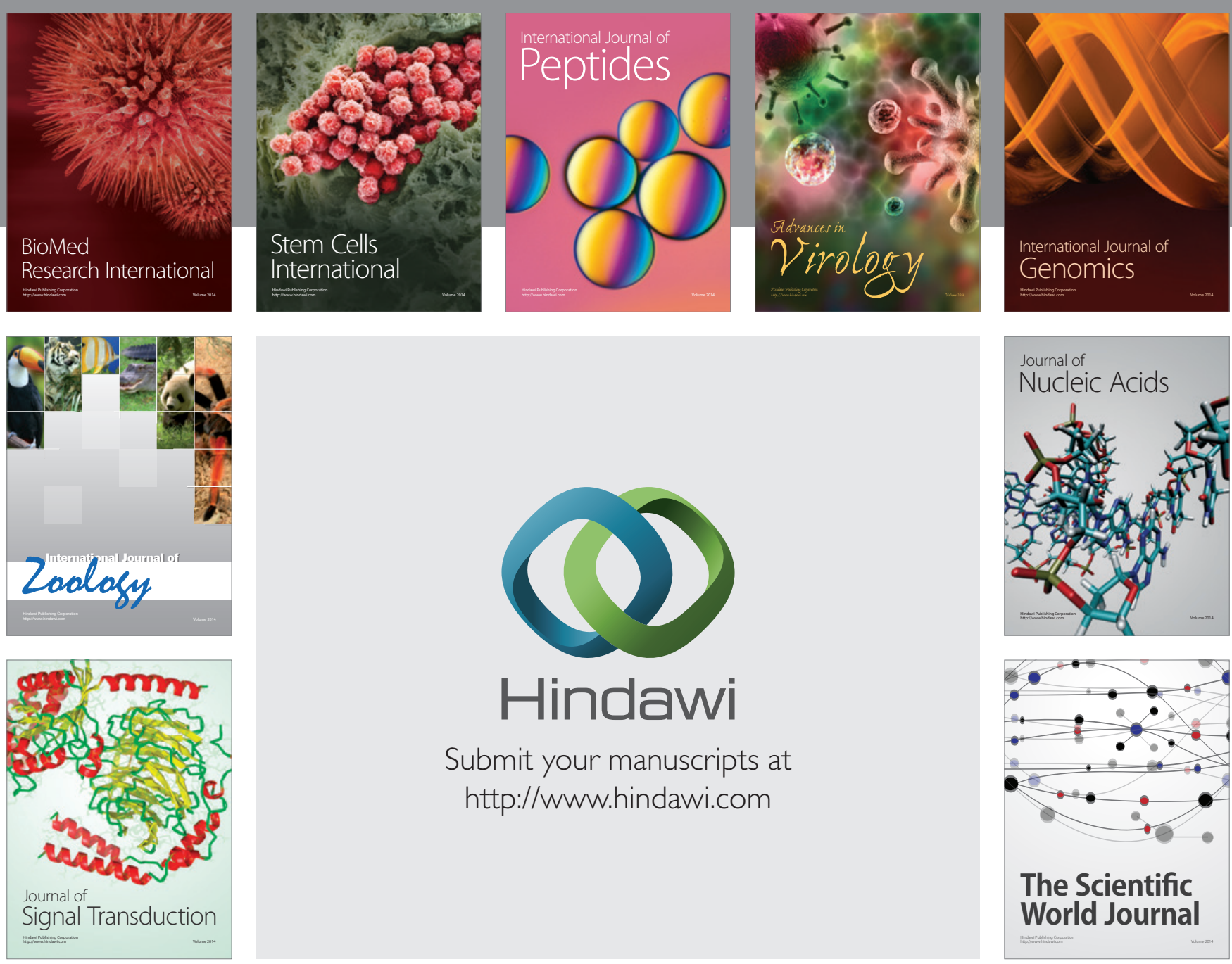

Submit your manuscripts at

http://www.hindawi.com
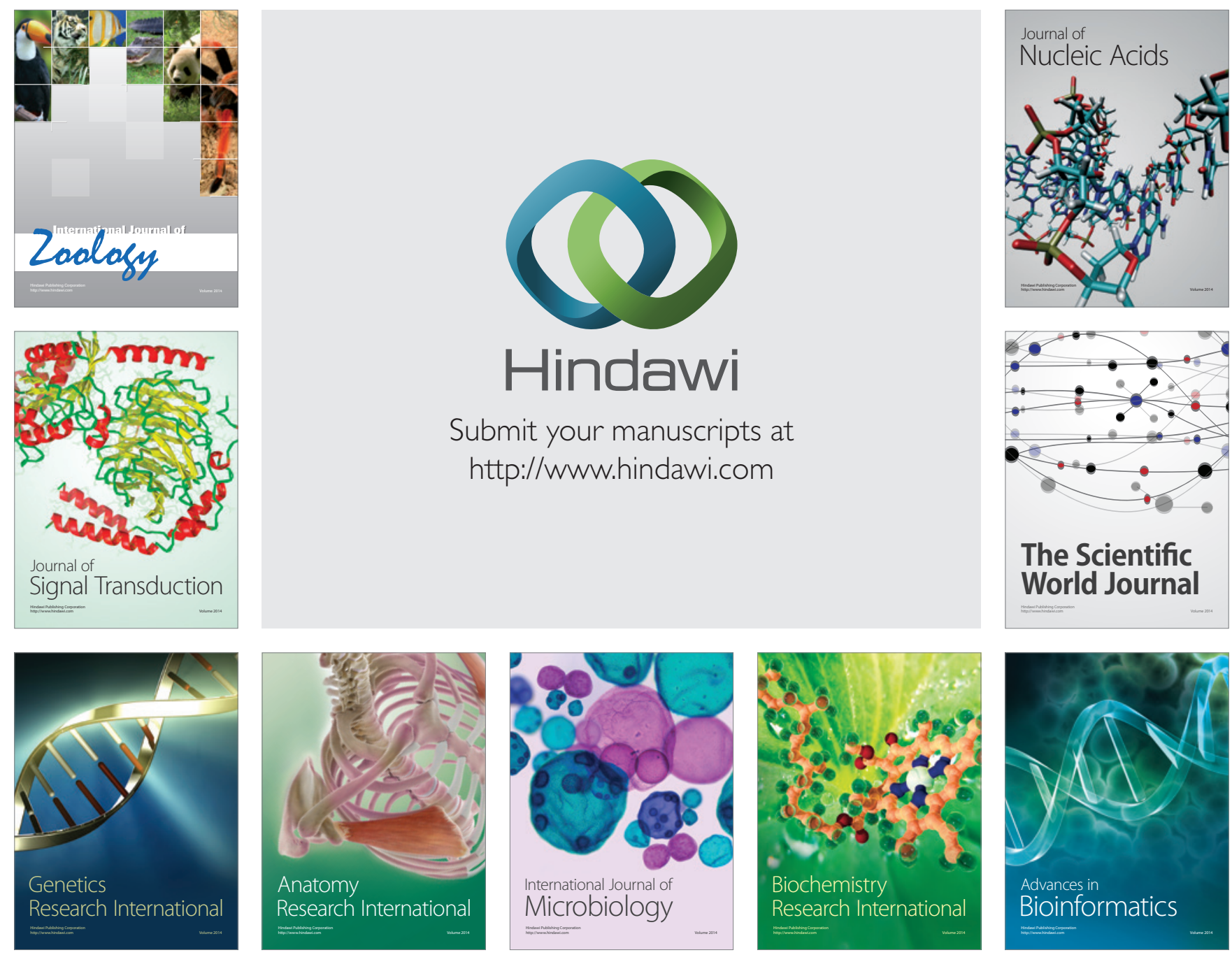

The Scientific World Journal
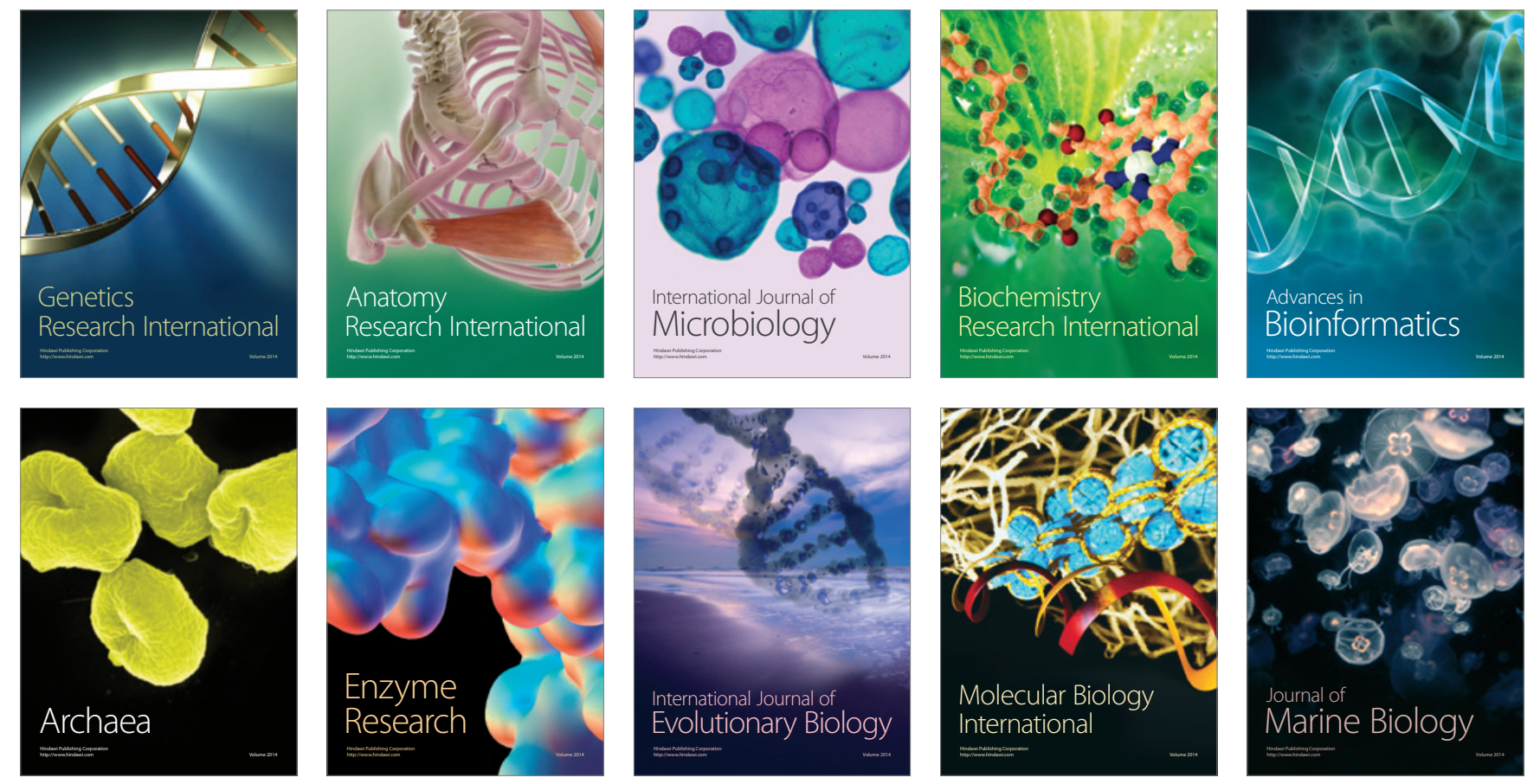